\title{
Real-Time 3D Analysis During Tomographic Experiments on tomviz
}

Jonathan Schwartz ${ }^{1}$, Chris Harris ${ }^{2}$, Jacob Pietryga ${ }^{1}$, Huihuo Zheng ${ }^{3}$, Prashant Kumar ${ }^{4}$, Anastasiia Visheratina ${ }^{4}$, Nicholas Kotov ${ }^{4}$, Yi Jiang ${ }^{5}$, Marcus Hanwell ${ }^{6}$ and Robert Hovden ${ }^{1}$

${ }^{1}$ Department of Materials Science and Engineering, University of Michigan, United States, ${ }^{2}$ Kitware, United States, ${ }^{3}$ Argonne Leadership Computing Facility, Argonne National Laboratory, United States, ${ }^{4}$ Department of Chemical Engineering, University of Michigan, United States, ${ }^{5}$ Advanced Photon Source, Argonne National Laboratory, Lemont, IL 60439, USA, United States, ${ }^{6}$ National Synchrotron Light Source II, Brookhaven National Laboratory, United States

Three-dimensional (3D) characterization across the nanoscale is now possible using scanning / transmission electron microscopes (S/TEM) [1-3]. Unfortunately, tomographic reconstructions can take one to several days to complete depending upon the dataset size or algorithm(s) employed. Even worse, the reconstruction occurs offline, long after all the data has been collected, preventing immediate interpretation during an ongoing experiment. While advancements in detector hardware have boosted throughput with digital data collection, substantial human effort and computational resources are still required to process the raw data [4]. Thus, it has been a longstanding goal to begin 3D analysis of specimens in real-time allowing immediate assessment of nanoscale structure [5]. Achieving high-throughput electron tomography requires an integrated pipeline that links the microscope hardware to optimized reconstruction algorithms and efficient 3D volumetric visualization. Moreover, this pipeline should be multi-threaded to run dynamic visualizations that updates as new data is collected or reconstruction algorithms proceed.

Here we present interactive 3D visualization that seamlessly runs while experimental projections are collected in an electron microscope using the tomviz platform (www.tomviz.org). Tomviz presents 3D materials or biological structure to scientists in real-time enabling high-throughput specimen interpretation with immediate tomogram visualization. Figure 1 illustrates the pipeline where projections are simultaneously reconstructed into an interactive 3D render. During experimental acquisition, tomviz monitors for new projections (Fig. 1a) which is continuously appended into the reconstruction process. After a reconstruction updates, tomviz immediately renders the 3D volume (Fig. 1b). Real-time tomography demands maximally efficient computational speed to ensure the optimization runs faster than the experimental data acquisition rate. Tomograms are reconstructed in parallel with data acquisition and a high-quality $3 \mathrm{D}$ reconstruction is available before the experiment ends (Fig. 1c).

We demonstrate real-time tomography on a helical nanoparticle comprised of a Cysteine amino-acid coordinated with Cadmium (Cys/Cd) [6]. These semiconducting nanoparticles contain strong tunable chiroptical properties due to its twisted geometry. Direct visualization of a specimen's 3D structure enables immediate identification of morphological and internal information shortly after a tomography experiment begins. As shown in Fig. 1b, the overall specimen morphology can be observed in as early as 5 minutes. The specimen's left-handed chirality is discerned in the first twenty minutes and a high-resolution volume is available within $50 \%$ of the experiment (20-30 minutes).

The multithreaded pipeline in tomviz enables interactive 3D analysis of the current reconstruction state with minimal impact on performance. A robust graphical interface (Fig. 2a) allows for 3D objects to be rendered as shaded contours or volumetric projections and these objects can be rotated, cropped, or sliced as the reconstruction evolves. Thus, scientists can go beyond superficial inspection to quantify specimen features or internal structure while simultaneously operating the microscope. This immediate feedback can save researchers days of effort as reconstructions are no longer processed offline. However, real-time tomography also improves offline analysis by dynamically visualizing iterative tomographic reconstructions as they progress. Tomviz now implements live visualization for several state-of-the-art algorithms including Simultaneous Iterative Reconstruction Technique (SIRT)[7] and Dynamic Compressed Sensing [8] which 
extends L1 regularization into a real-time framework. Whether the computation runs online or offline, tomviz users can evaluate 3D specimen structure and optimize the reconstruction accuracy in real-time.

Tomviz offers unparalleled electron tomography throughput with real-time 3D analysis — it is open-source and available to all institutions for download at www.tomviz.org

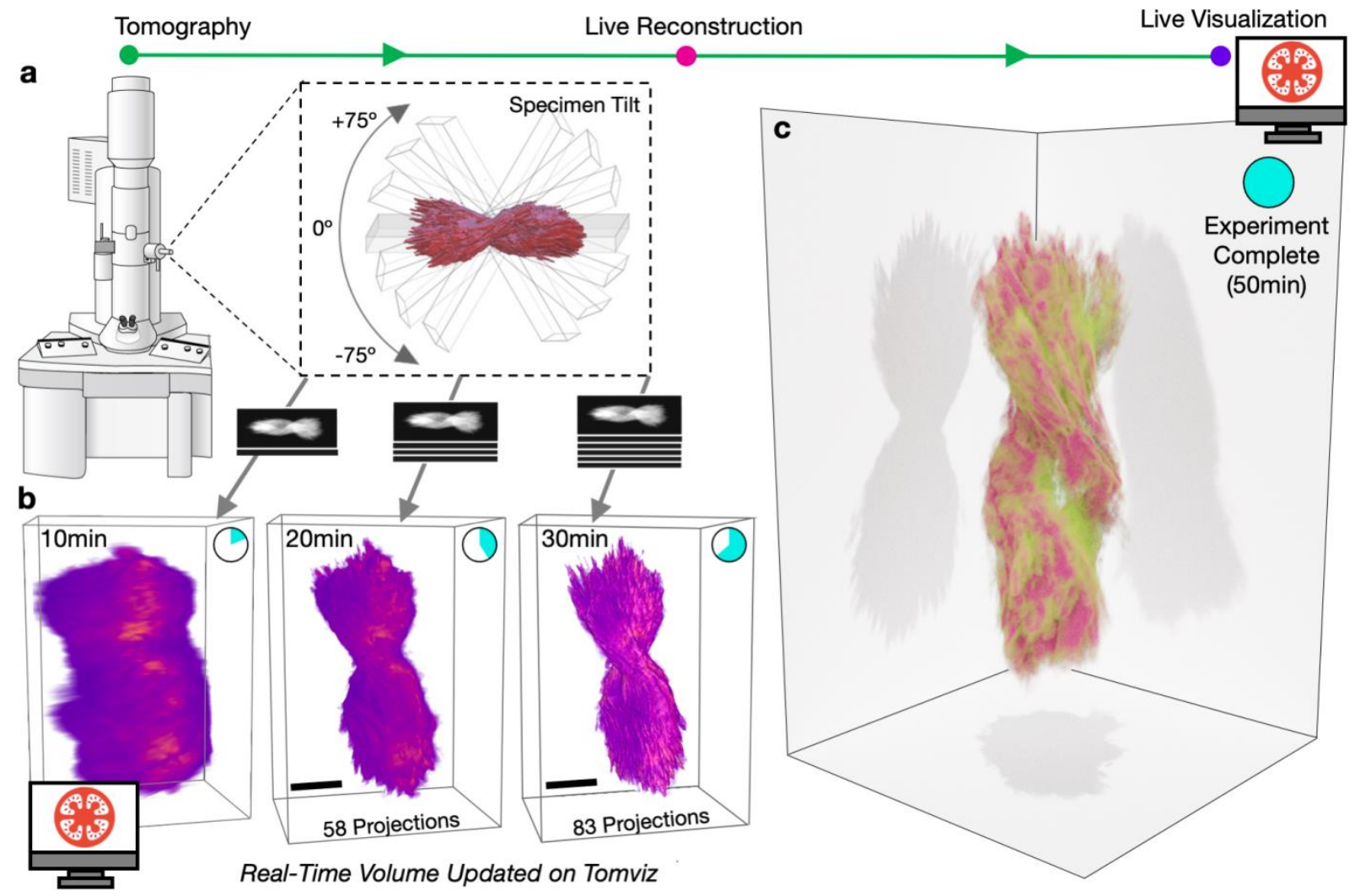

Figure 1. Figure 1: Real-Time tomography workflow of a helical nanoparticle with 3D tomograms visualized on tomviz. a, Specimen projections are sequentially collected in an electron microscope across an angular range $\left( \pm 75^{\circ}\right)$ and continually passed to tomviz for reconstruction and live $3 \mathrm{D}$ visualization. $\mathrm{b}$, As projections accumulate during the experiment, the reconstruction updates in real-time and the resolution improves. Scale bar, $100 \mathrm{~nm}$. c, A high-quality tomogram is available for data interpretation at or before the end of an experiment. 

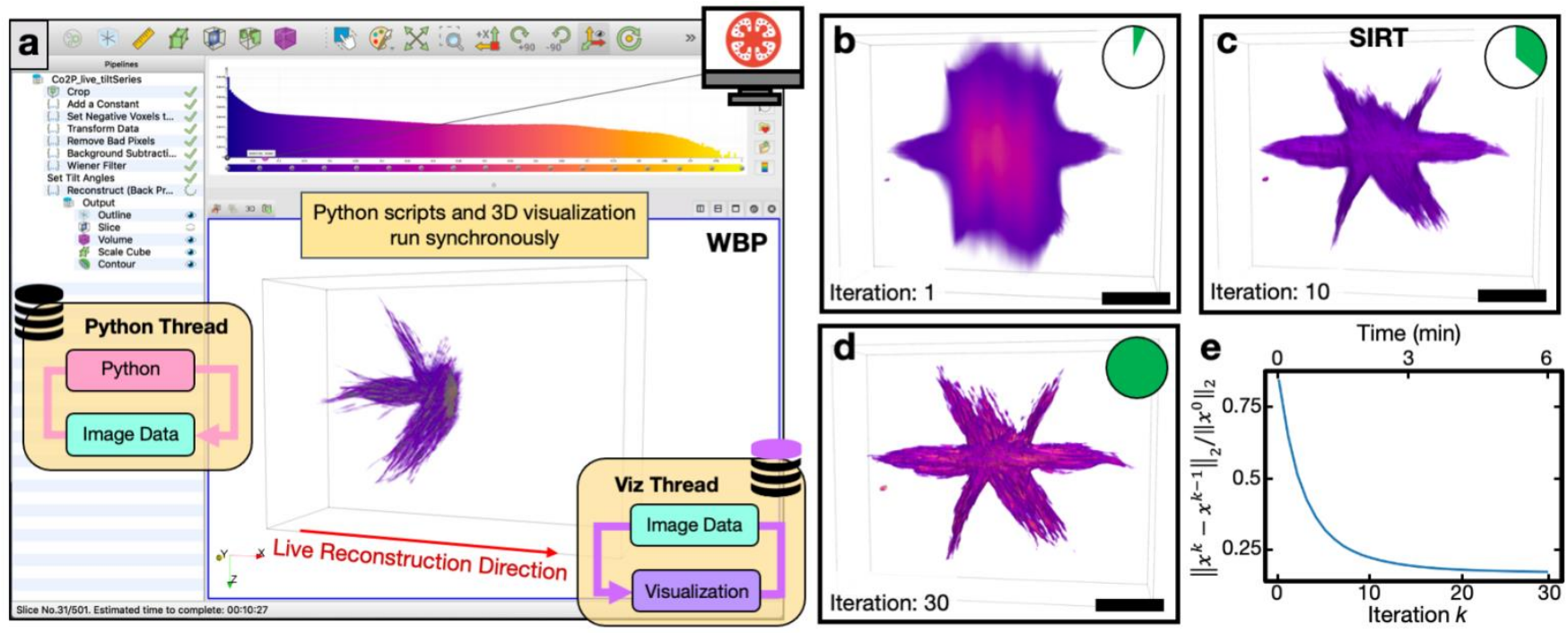

Figure 2. Figure 2: The tomviz GUI and real-time 3D visualization. a, Thetomviz platform is composed of a multithreaded pipeline that synchronously handles tomographic reconstruction and $3 \mathrm{D}$ visualization on separate threads. As tomographic reconstructions proceed, visualizations dynamically update and remain interactive for analysis. b-d, Visualization of the Co2P nanoparticle early, mid and end of a SIRT reconstruction process. The tomogram incrementally improves and sharp features become visible as iterations progress. The final iteration converges to a high-resolution tomogram representative of the specimen. Scale bar, 100nm. e, A plot of the normalized residual illustrates convergence.

\section{References}

[1] R. Crowther, L. Amos, J. Finch, D. Rosier, A. Klug, Nature226, 421 (1970).

[2] P. Midgley and M. Weyland. Ultramicroscopy96, 413 (2003).

[3] R. Hovden and D. Muller. MRS Bulletin45, 298 (2020).

[4] M. Chen, et. al. Nat. Methods16, 1161 (2019).

[5] M. Schorb, I. Haberbosch, H. Wim, Y. Schwab and D. Mastronarde. Nat. Methods16, 471 (2019).

[6] J. Yan, et. al. Chem. Of Mater.32, 476 (2019).

[7] P. Gilbert. J. Theor. Biol.36, 105 (1972).

[8] J. Schwartz, et. al. Ultramicroscopy219, 113122 (2020). 